\title{
TRAINING MODEL FOR PROMOTING TRANSLATION FROM RESEARCH TO CLINICAL SETTINGS: UNIVERSITY OF ALABAMA AT BIRMINGHAM TRAIN- ING FOR CONSTRAINT-INDUCED MOVEMENT THERAPY
}

\author{
David M. Morris, PT, PhD; Edward Taub, PhD
}

\section{INTRODUCTION}

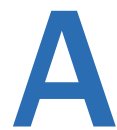
common challenge in stroke rehabilitation research is the translation of research findings into the clinical setting. Multiple factors present obstacles to dissemination and adoption, including the complexity of the physical rehabilitation protocols and the shift in practice required by treatment philosophies that require innovative approaches.

The UAB Train-

ing for CI Therapy

Workshop Program

was developed to

overcome the know-

ledge-to-action gap

apparent between

CI therapy research

and clinical practice.

The Constraint-Induced Movement $(\mathrm{Cl})$ Therapy Research Group (CITRG) at the University of Alabama at Birmingham (UAB) has developed and conducted a 5-day training program for clinicians and researchers that has been delivered semiannually since 2005. The goals of this training program are to accurately disseminate the $\mathrm{Cl}$ therapy protocol for upper-limb recovery after stroke that has been researched at $\mathrm{UAB}$, provide hands-on instruction, clarify questions and correct misunderstandings about components of the treatment protocol, and provide advice on implementing it in clinical and research settings. To date, more than 170 clinicians and researchers, from the United States and abroad, have participated in the training program at UAB. Many have successfully carried out the protocol back in their institutions. This JRRD guest editorial describes the evolution of the UAB CI Therapy Training Program, details the instructional elements provided in the program, describes posttraining experiences of selected program participants, and discusses future plans for the training program to further enhance the translation of $\mathrm{Cl}$ therapy into other clinical and research settings. The program provides an example of how research findings can be translated into a practical clinical program and then disseminated for use by clinicians and researchers.

\section{CHALLENGE OF RESEARCH TRANSLATION}

It is well known that advances in rehabilitation research face many obstacles to adoption in clinical practice. The Agency for Healthcare Research and Quality reports that it may take clinicians as long as 1 or 2 decades to incorporate original research into clinical practice [1]. In 2006, Graham and colleagues published an article discussing the slow, haphazard, and often incomplete transfer of findings from research into the clinical setting, calling this phenomenon a "knowledgeto-action gap" [2]. This delay creates a substantial obstacle to improving the quality of healthcare. A 2010 literature review concerning knowledge translation in healthcare further explores this issue by describing evolving models that have been proposed to integrate research into clinical practice [3]. Early models conceptualized a linear, unidirectional model of information sharing in which researchers simply reported their findings to clinicians in hopes that their innovations would be adopted into practice. This approach has been ineffective because it is too passive and fails to recognize the important cultural differences between the research and clinical communities and the resistance to making changes in established clinical practice. Later models called attention to these important cultural differences, yet did little to resolve the barriers to translation they create. The evidence-based medicine (EBM) movement of the 1990s aimed to increase the scientific rigor of clinical investigations and improve clinicians' ability to evaluate research publications before incorporating them into their clinical decision-making. While significantly enhancing the connection between the research and clinical communities, the EBM approach is still challenged by the lack of a tradition or sufficient reward for clinicians to publish and disseminate research findings in 
a timely fashion, difficulties faced by clinicians in interpreting the results presented in research publications, misunderstandings encountered when attempting to implement multicomponent and complex protocols that are inadequately described because of space limitations applied to manuscripts by most journals, and the remaining cultural differences that exist between the research and clinical communities. More recent models attempting to close the knowledge-to-action gap recommend more active engagement on the part of clinicians, as opposed to being passive recipients of information [3]. They call for a heightened process of interaction and collaboration between researchers and clinicians so that cultural barriers can be identified and overcome. To assure proper transfer, this two-way exchange process should be ongoing, with long-term relationships established between researchers and clinicians [3].

The case of $\mathrm{Cl}$ movement therapy appears to be a good example of a knowledge-to-action gap. $\mathrm{Cl}$ therapy has been shown to be an efficacious intervention for improving limb use following neurologic injury in numerous clinical trials, supported by systematic reviews of the literature and professional practice guidelines [4-18]. However, the approach has not been widely applied to routine clinical practice [19]. Evidence suggests that both clinicians and patients express concern about its clinical feasibility and that they poorly understand the intervention protocol [20-23]. To add to their confusion, even the scientific literature inconsistently describes the approach. For example, an unpublished review of 28 randomized controlled trials reporting use of $\mathrm{Cl}$ therapy indicated that only three of the studies used the full $\mathrm{Cl}$ therapy protocol as described by the originators of the approach. The most commonly omitted elements of the recommended protocol, the components of the transfer package, are the most effective [16-18]. Since 2005, the CITRG at UAB has conducted a 5-day training program for clinicians and researchers in an attempt to resolve the knowledge-toaction gap concerning $\mathrm{Cl}$ therapy for improving upperlimb function following stroke in adults. The workshops are usually held semiannually, and in 2009, a pediatric $\mathrm{Cl}$ therapy segment was added to one workshop each year. While feedback from training participants has been overwhelmingly positive, no formal research endeavors have been attempted to examine how effectively training participants are using the protocol back in their clinical settings. Still, we believe that sharing our experiences will be useful to other researchers wishing to close the knowledge-to-action gap through training activities.

\section{CONSTRAINT-INDUCED MOVEMENT THERAPY PROTOCOL}

$\mathrm{Cl}$ therapy is a rehabilitation approach that multiple studies have shown to substantially enhance hemiparetic arm motor recovery in stroke survivors [4-18,24]. The approach has also been successfully applied to the treatment of other diagnostic groups (e.g., children with cerebral palsy [25-26], adults with traumatic brain injury [27-28], adults with multiple sclerosis [29]) and to other functional skills (e.g., ambulation [9], speech [30-31]) $[6,9,17]$. The most prominent outcome of the signature intervention for upper-limb hemiparesis is a very large increase in actual use of the paretic arm in daily life.

This family of interventions was developed by our UAB research group, which is directed by Edward Taub, $\mathrm{PhD}$. The approach is derived directly from an extensive program of basic research with deafferented monkeys conducted by Taub (reviewed in Taub [32-33]). The signature $\mathrm{Cl}$ therapy protocol is multicomponent and consists of four broad categories of techniques: (1) supervised, repetitive task-oriented motor training of the more-affected arm; (2) training by means of the behavioral technique "shaping"; (3) limiting use of the less-affected arm for many hours daily; and (4) use of adherence-enhancing behavioral strategies to promote more-affected arm use in the home and community (the transfer package) [13,16-18].

$\mathrm{Cl}$ therapy differs substantially from traditional rehabilitation approaches and therefore calls for a significant paradigm shift in clinicians' thinking about and practice of physical rehabilitation [11]. First, there is a focus on promoting recovery of the more-affected arm by taking advantage of neuroplasticity and intense concentrated practice using a shaping procedure. Second, during training a greater emphasis is placed on using the more-affected arm to accomplish a large number of functionally relevant tasks rather than on improving the quality of the movement per se, which is the traditional approach. Third, explicit attention and specific behavioral procedures, which are part of the adherence-enhancing transfer package, are directed toward increasing use of the more-affected arm in daily life 
[13,17-18]. Clinicians have not ignored the latter treatment approach previously; however, the techniques that were employed were not applied systematically and did not advance much beyond recommendations given in the clinic. Although our laboratory's protocols have been published [5,13-14,17-18], the difficulty of translating the different perspective and approach demanded by $\mathrm{Cl}$ therapy into practice is apparent in the wide range of procedures employed (and not employed) and the outcomes obtained in published studies involving $\mathrm{Cl}$ therapy.

The amount of $\mathrm{Cl}$ therapy research for stroke and cerebral palsy in the peer-reviewed literature has grown at a increasing rate since the first study appeared in 1993, 21 years ago. In the first 5 years, there were just four studies. A recent analysis done by a member of our research group (V. Mark) suggests an unforeseen and profuse growth in both the number of $\mathrm{Cl}$ therapy trials as well as review papers. Considering adult $\mathrm{Cl}$ therapy research, there are $\mathbf{2 7 6}$ trials to date and $\mathbf{2 2 5}$ review papers. For the past 10 years, the combined average publication rate has been 43 reports a year. In addition, there have been more than 70 reports of pediatric $\mathrm{Cl}$ therapy research to date. There also has been a consistent and important increase in the diffusion of $\mathrm{Cl}$ therapy, as evidenced by the growth in the number of new research laboratories that have tested $\mathrm{Cl}$ therapy and published their findings in the peerreviewed literature. There have altogether been 130 different laboratories, representing 29 countries, that have published adult and pediatric $\mathrm{Cl}$ therapy research. Despite significant support from the research community, clinical adoption of $\mathrm{Cl}$ therapy remains relatively slow [19].

\section{University of Alabama at Birmingham Training for Constraint-Induced Movement Therapy}

Laboratories that have used our complete, published protocol; whose clinicians trained in our laboratory; and, in three cases, were monitored periodically by one of us (E. Taub), obtained results that were similar to ours [7-8,34-35]. Other laboratories that do not use the full protocol generally get positive treatment effects, but they are substantially reduced, especially for spontaneous use of the more-affected upper limb in the life situation. The usual differences between studies with strongly positive results and studies with reduced effects is that the transfer package techniques are omitted; there is also often no mention of shaping in the studies with reduced effects. To help disseminate the $\mathrm{Cl}$ therapy approach beyond $\mathrm{UAB}$ and address the misconceptions and misunderstanding about its implementation, thereby improving clinical outcomes, we established the UAB Training for $\mathrm{Cl}$ Therapy Workshop Program in 2005. In the years before the initiation of the training program, our laboratory was receiving an increasing number of requests from researchers and clinicians for visits to our laboratory to observe and learn our research protocol. While such visits can be stimulating, they are time-consuming and disruptive to the regular duties of any research or clinic group. In part, the training program was developed to address these requests more efficiently. It seemed more reasonable to disrupt the operations of the research group with a major training effort for only 2 weeks each year (two separate training programs) as opposed to more frequent shorter visits. This program follows the model of the training workshop hosted at UAB for clinical sites participating in the Extremity Constraint-Induced Therapy Evaluation (EXCITE) trial. EXCITE was one of the first multisite national trials in physical rehabilitation; UAB served as the training center for the trial $[14,36]$. The current 5-day intensive training program for rehabilitation professionals includes didactic and hands-on experiences to promote understanding of the rationale and mechanics of performing the $\mathrm{Cl}$ therapy protocol procedures and their theoretical and data bases. The program presents a substantial amount of didactic background material (e.g., history, theoretical basis, protocol components) and calls for a significant shift in participants' treatment philosophy in a short period of time. Instructional activities include lectures, discussions, demonstrations, and opportunities to practice techniques with individuals recovering from stroke under the supervision of members of the CITRG. A detailed workshop manual is provided for participants and includes selected research articles and detailed descriptions of testing and training procedures. DVDs containing videos demonstrating the correct methods for administering selected $\mathrm{Cl}$ therapy treatment and testing procedures are also provided. The program focuses on $\mathrm{Cl}$ therapy treatment for upper-limb rehabilitation for adults with chronic stroke and children with cerebral palsy. The course is designed for application by rehabilitation professionals working in ambulatory settings (e.g., outpatient clinics, home health) and the training program's target audience is primarily physical therapists, occupational therapists, physical 
therapist assistants, and certified occupational therapist assistants with a desire to develop $\mathrm{Cl}$ therapy programs in their clinical setting. However, other health professionals (e.g., psychologists, physicians, chiropractors), researchers, and individuals from inpatient facilities have also participated in the training program. Before attending, participants are expected to possess a basic understanding and competency with physical rehabilitation strategies for patients recovering from neurologic injury. The course content and materials are presented in English. It has been important to emphasize this in our descriptive literature, since we have had many international participants enroll in the program.

To date, the program for the treatment of adults has been offered 12 times at UAB, with approximately 170 participants completing the program. The Table presents the countries represented by participants and their professional titles. A separate track addressing an upper-limb protocol for children with cerebral palsy has also been offered four times. The program has been well received by participants, with consistently high ratings on course evaluations. The training program has also been conducted on request internationally on four occasions (Sydney, Australia, in 2010; Belfast, Ireland, in 2011; Singapore in 2011; and Ostrava, Czech Republic, in 2012). The course delivered at the University of Ulster in Ireland was part of postprofessional coursework for the Masters of Science in Allied Health Sciences Program. The request for these international offerings indicates a substantial interest in $\mathrm{Cl}$ therapy outside of the United States.

\section{Experiences of Selected Participants}

\section{Sydney, Australia}

Phillip Fay, OT, attended the training program in June 2009. Since that time, he has implemented the full $\mathrm{Cl}$ therapy protocol with adults recovering from stroke and brain injury in a large institutional setting as well as a private practice clinic. To date, the protocol has been used with 31 participants, with an average improvement of 1.84 on the Amount Scale of the Motor Activity Log (MAL). These results approach those observed by the UAB research group. He has also paired the $\mathrm{Cl}$ therapy protocol with other rehabilitation strategies (e.g., prism adaptation therapy, sensory retraining, specific shoulder exercises) with good success. He has given several presentations about $\mathrm{Cl}$ therapy and his experiences for therapists across Australia.

\section{Belfast, Ireland}

Katy Pedlow, PT, PhD, attended the training program in October 2009. Since then, she has initiated a variety of $\mathrm{Cl}$ therapy projects, including some associated with her doctoral studies at the University of Ulster. In addition to conducting an online survey of therapists concerning use of $\mathrm{Cl}$ therapy in the United Kingdom, she organized a clinical feasibility randomized clinical trial (RCT) with 21 participants in a regional acquired brain injury treatment unit. In this study, participants were randomized to receive a $\mathrm{Cl}$ therapy protocol or a Bobath-based intervention program [37]. A publication describing results is expected soon. Additional studies associated with the RCT included a qualitative study of experiences of therapists delivering the $\mathrm{Cl}$ therapy protocol, a behavioral mapping study of the therapists' activities as they used the $\mathrm{Cl}$ therapy protocol, and standardization procedures carried out collaboratively with the UAB CITRG to assure therapist fidelity to the study protocol. Dr. Pedlow has given six poster presentations and seven invited presentations and has written three grant proposals concerning the use of $\mathrm{Cl}$ therapy with adults with brain injury.

\section{São Paulo, Brazil}

The Association for Assistance to Deficient Children (AACD) is a private, nonprofit organization established since 1950 in Brazil to promote the welfare of all people with physical disabilities. AACD sent three therapists to the October 2009 training program and four additional therapists to the October 2012 training program. These therapists, along with their colleagues at AACD, have established $\mathrm{Cl}$ therapy treatment programs for children and adults with stroke, cerebral palsy, traumatic brain injury, spinal cord injury, and brain tumors. To date, they have provided $\mathrm{Cl}$ therapy treatment to approximately 70 adults and 55 children. The team has observed change scores on the Amount Scale of the MAL of 1.8 and 2.6 for pediatric and adult participants, respectively. Therapists at AACD have translated into Portuguese outcome measures developed at UAB and, in collaboration with the UAB team, have explored the reliability, validity, and sensitivity of the translated instruments. They have also established $\mathrm{Cl}$ therapy research projects exploring the clinical application of 
Cl therapy in São Paulo. The São Paulo Cl therapy team has also conducted many training programs for Brazilian therapists and physical therapy and occupational therapy students. AACD Is planning a large scale $\mathrm{Cl}$ therapy conference in Brazil in March 2014.

\section{Future Directions for Training Program}

The UAB Training for $\mathrm{Cl}$ Therapy Workshop Program was developed to overcome the knowledge-toaction gap apparent between $\mathrm{Cl}$ therapy research and clinical practice. Since beginning in 2005 , the training team has modified the training program based on feedback from participants. For example, the training team added a hands-on practice session in which participants apply protocol components on each other before the patient models arrive for further practice. This additional practice session allows the training participants to ask detailed questions and resolve misunderstandings before applying the techniques with patients with stroke who previously received $\mathrm{Cl}$ therapy and are paid to participate. It also dramatically enhances participants' confidence with the protocol. The training manual has also been modified several times by adding requested materials as well as removing unnecessary documents. The strongly positive responses from training participants and examples of successful integration of $\mathrm{Cl}$ therapy methods into clinical practice suggest that the training program has had a useful effect. The training staff attributes the program's effectiveness largely to the opportunities for active therapeutic engagement that are provided (i.e., supervised treatment activities using $\mathrm{Cl}$ therapy techniques with people with stroke) and to the in-depth two-way communication between the research staff and participants. However, the current training program is carried out in a single location, which is often at a considerable distance from the participants' home location; therefore, they must incur travel and living expenses and lose work time to attend the workshop. These factors limit the program from reaching a large number of clinicians and in establishing the long-term collaborative relationship that is advocated by contemporary knowledge-to-action models. A Web-based training format could make participation feasible for a larger audience, especially for international participants. Using such a format, the didactic portion of the program could be converted into self-paced, online training modules. These modules may be superior to live lectures, in that more active learning strategies could be employed and self-assessment tools could be incorporated to periodically assess participants' understanding of the concepts presented. Periodic live chats and/or a discussion board could be used for promoting interaction between the research staff and participants, providing opportunities for exploring questions and correcting misperceptions. Following the completion of the formal training program, participants could stay engaged by way of an online " $\mathrm{Cl}$ therapy community of practice" using online communication (e.g., discussion boards, email lists) for idea sharing, triage for clinical challenges encountered, and dissemination of new $\mathrm{Cl}$ therapy research.

The biggest challenge faced by going online with the training would be the inability to provide the supervised hands-on practice opportunities in which training participants actually employ the protocol techniques with persons recovering from stroke. Many participants cite this part of the training program as being the most beneficial. Potential substitutes for the supervised practice could be structured laboratory assignments that could be completed independently by participants in their own clinical settings; these activities could be videotaped and shared by a videoconferencing application with the training program staff and with other participants for structured assessment. These activities could take the form of the standardization procedures used for the EXCITE multisite RCT of Cl therapy [36]. The research group also plans to develop similar training programs to disseminate other $\mathrm{Cl}$ therapy protocols (i.e., lower-limb function, aphasia) that have been developed and researched at UAB.

\section{CONCLUSIONS}

The term "knowledge-to-action gap" has been used to describe the slow and often incomplete translation of research findings into clinical practice. Recent publications have proposed better models that promote more active engagement by trainees and ongoing clinicianresearcher collaborations to facilitate faster and more accurate incorporation of research findings into clinical decision making. The UAB Training for $\mathrm{Cl}$ Therapy Workshop Program has been directed toward disseminating 
$\mathrm{Cl}$ therapy research findings and clinical procedures to a significant number of clinicians and researchers worldwide. Future enhancements to the training program are expected to result in enabling it to reach a larger audience.

\section{David M. Morris, PT, PhD; ${ }^{1 *}$ Edward Taub, PhD $^{2}$}

Departments of ${ }^{1}$ Physical Therapy and ${ }^{2}$ Psychology, University of Alabama at Birmingham, AL

*Email: morrisd@uab.edu

\section{REFERENCES}

1. Agency for Healthcare Research and Quality. Translating research Into practice (TRIP)-II: Fact sheet [Internet]. Rockville (MD): Agency for Healthcare Research and Quality; 2001. Available from http//www.ahrq.gov/research/findings/factsheets/translating/tripfac/index.html

2. Graham ID, Logan J, Harrison MB, Straus SE, Tetroe J, Caswell W, Robinson N. Lost in knowledge translation: Time for a map? J Contin Educ Health Prof. 2006;26(1): 13-24. [PMID:16557505]

http://dx.doi.org/10.1002/chp.47

3. Oborn E, Barrett M, Racko G. Working paper series. Knowledge translation in healthcare: A review of the literature. Cambridge (UK): Cambridge Judge Business School; 2010.

4. Taub E, Miller NE, Novack TA, Cook EW 3rd, Fleming WC, Nepomuceno CS, Connell JS, Crago JE. Technique to improve chronic motor deficit after stroke. Arch Phys Med Rehabil. 1993;74(4):347-54. [PMID:8466415]

5. Morris DM, Crago JE, Deluca SC, Pidikiti RD, Taub E. Constraint-induced movement therapy for motor recovery after stroke. NeuroRehabilitation. 1997;9(1):29-43. [PMID:24526089]

http://dx.doi.org/10.1016/S1053-8135(97)00012-7

6. Taub E, Crago JE, Uswatte G. Constraint-induced movement therapy: A new approach to treatment in physical rehabilitation. Rehabil Psychol. 1998;43:152-70. http://dx.doi.org/10.1037/0090-5550.43.2.152

7. Miltner WH, Bauder H, Sommer M, Dettmers C, Taub E. Effects of constraint-induced movement therapy on patients with chronic motor deficits after stroke: A replication. Stroke. 1999;30(3):586-92. [PMID:10066856] http://dx.doi.org/10.1161/01.STR.30.3.586

8. Kunkel A, Kopp B, Müller G, Villringer K, Villringer A, Taub E, Flor H. Constraint-induced movement therapy for motor recovery in chronic stroke patients. Arch Phys Med Rehabil. 1999;80(6):624-28. [PMID:10378486] http://dx.doi.org/10.1016/S0003-9993(99)90163-6

9. Taub E, Uswatte G, Pidikiti RD. Constraint-Induced Movement Therapy: A new family of techniques with broad application to physical rehabilitation-A clinical review. J Rehabil Res Dev. 1999;36(3):237-51. [PMID:10659807]

10. Taub E, Morris DM. Constraint-induced movement therapy to enhance recovery after stroke. Curr Atheroscler Rep. 2001;3(4):279-86. [PMID:11389792] http://dx.doi.org/10.1007/s11883-001-0020-0

11. Morris DM, Taub E. Constraint-induced therapy approach to restoring function after neurological injury. Top Stroke Rehabil. 2001;8(3):16-30.

[PMID:14523735] http://dx.doi.org/10.1310/BLJX-M89N-PTPY-JDKW

12. Taub E, Uswatte G, King DK, Morris D, Crago JE, Chatterjee A. A placebo-controlled trial of constraintinduced movement therapy for upper extremity after stroke.Stroke. 2006;37(4):1045-49.[PMID:16514097] http://dx.doi.org/10.1161/01.STR.0000206463. 66461.97

13. Morris DM, Taub E, Mark VW. Constraint-induced movement therapy: Characterizing the intervention protocol. Eura Medicophys. 2006;42(3):257-68. [PMID:17039224]

14. Winstein CJ, Miller JP, Blanton S, Taub E, Uswatte G, Morris D, Nichols D, Wolf S. Methods for a multisite randomized trial to investigate the effect of constraint-induced movement therapy in improving upper extremity function among adults recovering from a cerebrovascular stroke. Neurorehabil Neural Repair. 2003;17(3):137-52. [PMID:14503435] http://dx.doi.org/10.1177/0888439003255511

15. Wolf SL, Winstein CJ, Miller JP, Taub E, Uswatte G, Morris D, Giuliani C, Light KE, Nichols-Larsen D; EXCITE Investigators. Effect of constraint-induced movement therapy on upper extremity function 3 to 9 months after stroke: The EXCITE randomized clinical trial. JAMA. 2006;296(17):2095-2104. [PMID:17077374] http://dx.doi.org/10.1001/jama.296.17.2095

16. Gauthier LV, Taub E, Perkins C, Ortmann M, Mark VW, Uswatte G. Remodeling the brain: Plastic structural brain changes produced by different motor therapies after stroke. Stroke. 2008;39(5):1520-25. [PMID:18323492] http://dx.doi.org/10.1161/STROKEAHA.107.502229

17. Taub E. The behavior-analytic origins of constraint-induced movement therapy: An example of behavioral neurorehabilitation. Behav Anal. 2012;35(2):155-78. [PMID:23449867]

18. Taub E, Uswatte G, Mark VW, Morris DM, Barman J, Bowman MH, Bryson C, Delgado A, Bishop-McKay S. Method for enhancing real-world use of a more affected arm in chronic stroke: Transfer package of constraintinduced movement therapy. Stroke. 2013;44(5):138388. [PMID:23520237] http://dx.doi.org/10.1161/STROKEAHA.111.000559

19. Natarajan P, Oelschlager A, Agah A, Pohl PS, Ahmad SO, Liu W. Current clinical practices in stroke rehabilitation: Regional pilot survey. J Rehabil Res Dev. 2008;45(6): 
841-50. [PMID:19009470]

http://dx.doi.org/10.1682/JRRD.2007.04.0057

20. Page SJ, Levine P, Sisto S, Bond Q, Johnston MV. Stroke patients' and therapists' opinions of constraint-induced movement therapy. Clin Rehabil. 2002;16(1):55-60. [PMID:11837526] http://dx.doi.org/10.1191/0269215502cr473oa

21. Pedlow K, Lennon S, Wilson C. Application of constraintinduced movement therapy in clinical practice: an online survey. Arch Phys Med Rehabil. 2014;95:278-82.

22. Viana R, Teasell R. Barriers to the implementation of constraint-induced movement therapy into practice. Top Stroke Rehabil. 2012;19(2):104-14. [PMID:22436358] http://dx.doi.org/10.1310/tsr1902-104

23. Daniel L, Howard W, Braun D, Page SJ. Opinions of constraint-induced movement therapy among therapists in southwestern Ohio. Top Stroke Rehabil. 2012;19(3): 268-75. [PMID:22668681] http://dx.doi.org/10.1310/tsr1903-268

24. Nijland R, Kwakkel G, Bakers J, van Wegen E. Constraintinduced movement therapy for the upper paretic limb in acute or sub-acute stroke: A systematic review. Int J Stroke. 2011;6(5):425-33. [PMID:21951408] http://dx.doi.org/10.1111/j.1747-4949.2011.00646.x

25. Taub E, Ramey SL, DeLuca S, Echols K. Efficacy of constraint-induced movement therapy for children with cerebral palsy with asymmetric motor impairment. Pediatrics. 2004;113(2):305-12. [PMID:14754942] http://dx.doi.org/10.1542/peds.113.2.305

26. Taub E, Griffin A, Uswatte G, Gammons K, Nick J, Law CR. Treatment of congenital hemiparesis with pediatric constraint-induced movement therapy. J Child Neurol. 2011;26(9):1163-73. [PMID:21771948] http://dx.doi.org/10.1177/0883073811408423

27. Shaw SE, Morris DM, Uswatte G, McKay S, Meythaler JM, Taub E. Constraint-induced movement therapy for recovery of upper-limb function following traumatic brain injury. J Rehabil Res Dev. 2005;42(6):769-78. [PMID:16680614] http://dx.doi.org/10.1682/JRRD.2005.06.0094

28. Morris DM, Shaw SE, Mark VW, Uswatte G, Barman J, Taub E. The influence of neuropsychological characteristics on the use of $\mathrm{Cl}$ therapy with persons with traumatic brain injury. NeuroRehabilitation. 2006;21(2): 131-37. [PMID:16917159]

29. Mark VW, Taub E, Bashir K, Uswatte G, Delgado A, Bowman MH, Bryson CC, McKay S, Cutter GR. ConstraintInduced Movement therapy can improve hemiparetic progressive multiple sclerosis. Preliminary findings. Mult Scler. 2008;14(7):992-94. [PMID:18573826] http://dx.doi.org/10.1177/1352458508090223

30. Pulvermüller F, Neininger B, Elbert T, Mohr B, Rockstroh B, Koebbel P, Taub E. Constraint-induced therapy of chronic aphasia after stroke. Stroke. 2001;32(7):1621-26.
[PMID:11441210]

http://dx.doi.org/10.1161/01.STR.32.7.1621

31. Johnson ML, Taub E, Harper LH, Wade JT, Bowman MH, Bishop-McKay S, Haddad MM, Mark VW, Uswatte G. An enhanced protocol for CI Aphasia therapy: CIAT II-A case series. Am J Speech Lang Pathol. 2013 Sep 9. Epub ahead of print. [PMID:24018698]

32. Taub E. Movement in nonhuman primates deprived of somatosensory feedback. Exerc Sport Sci Rev. 1976;4: 335-74. [PMID:828579]

http://dx.doi.org/10.1249/00003677-197600040 $-00012$

33. Taub E. Somatosensory deafferentation research with monkeys: implications for rehabilitation medicine. In: Ince LP, editor. Behavioral psychology in rehabilitation medicine: Clinical applications. New York (NY): Williams \& Wilkins; 1980. p. 371-401.

34. Sterr A, Elbert T, Berthold I, Kölbel S, Rockstroh B, Taub E. Longer versus shorter daily constraint-induced movement therapy of chronic hemiparesis: An exploratory study. Arch Phys Med Rehabil. 2002;83(10):1374-77. [PMID:12370871] http://dx.doi.org/10.1053/apmr.2002.35108

35. Dettmers C, Teske U, Hamzei F, Uswatte G, Taub E, Weiller C. Distributed form of constraint-induced movement therapy improves functional outcome and quality of life after stroke. Arch Phys Med Rehabil. 2005; 86(2):204-9. [PMID:15706544] http://dx.doi.org/10.1016/j.apmr.2004.05.007

36. Morris DM, Taub E, Macrina DM, Cook EW, Geiger BF. A method for standardizing procedures in rehabilitation: Use in the extremity constraint induced therapy evaluation multisite randomized controlled trial. Arch Phys Med Rehabil. 2009;90(4):663-68. [PMID:19345784] http://dx.doi.org/10.1016/j.apmr.2008.09.576

37. Howie J. Neuro-developmental treatment approach: Theoretical foundations and principles for clinical practice. Laguna Beach (CA): Neuro-Developmental Treatment Association; 2004. p. 38.

This article and any supplementary material should be cited as follows:

Morris DM, Taub E. Training model for promoting translation from research to clinical settings: University of Alabama at Birmingham training for constraint-induced movement therapy. J Rehabil Res Dev. 2014;51(2):xi-xviii.

http://dx.doi.org/10.1682/JRRD.2014.01.0008

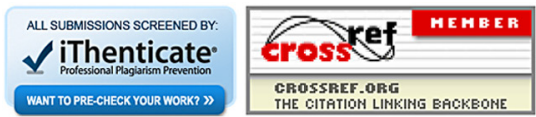

